\title{
Effects of Aging and Cyclosporin A on Collagen Turnover in Human Gingiva
}

\author{
N. Gagliano ${ }^{1 *}$, F. Costa ${ }^{1}$, G.M. Tartaglia ${ }^{1}$, L. Pettinari ${ }^{1}$, F. Grizzi ${ }^{2}$, C. Sforza ${ }^{1}$, N. Portinaro ${ }^{3}$, \\ M. Gioia ${ }^{1}$ and G. Annoni ${ }^{4}$
}

\author{
${ }^{I}$ Department of Human Morphology and Biomedical Sciences "Città Studi", EML-Extracellular Matrix Laboratory, \\ Università degli Studi di Milano, School of Medicine, Milan, Italy \\ ${ }^{2}$ Laboratories of Quantitative Medicine, Istituto Clinico Humanitas IRCCS, Rozzano, Milan, Italy \\ ${ }^{3}$ Department of Pediatric Orthopaedic Surgery, Istituto Clinico Humanitas, Rozzano, Milan, Italy \\ ${ }^{4}$ Department of Internal Medicine and Prevention and Geriatric Unit, S.Gerardo Hospital, University of Milano- \\ Bicocca, Milan, Italy
}

\begin{abstract}
:
Background: We aimed at characterizing the aging gingiva analyzing: i) collagen content and turnover in human gingival tissues and fibroblasts obtained from healthy young and aging subjects. ii) the effect of cyclosporin A administration in human cultured gingival fibroblasts obtained from aging compared to young subjects.

Methods: Morphological analysis was performed on haematoxylin-eosin and Sirius red stained paraffin-embedded gingival biopsies from young and aging healthy subjects. The expression of the main genes and proteins involved in collagen turnover were determined by real time PCR, dot blot and SDS-zymography on cultured young and aging gingival fibroblasts, and after cyclosporin A administration.

$\underline{\text { Results: }}$ Our results suggest that in healthy aged people, gingival connective tissue is characterized by a similar collagen content and turnover. Collagen turnover pathways are similarly affected by cyclosporin A treatment in young and aging gingival fibroblasts.

Conclusions: Cyclosporin A administration affects gingival collagen turnover pathways in young and aging fibroblasts at the same extent, suggesting that during aging cyclosporin A administration is not related to relevant collagen turnover modifications.
\end{abstract}

Keywords: Aging, gingiva, collagen turnover, matrix metalloproteinases, SPARC, cyclosporin A, gingival overgrowth.

\section{INTRODUCTION}

The oral cavity is formed by different specialized tissues that synergistically concur at least in three essential physiological functions in humans: phonation, nutrition, and defence of the host against a variety of pathogens, i.e. xenobiotics, bacteria, viruses and mycetes. In healthy aging, these tasks are modestly affected, but oral health declines, possibly due to the age-related ability of the different tissues to maintain homeostasis. In fact, the oral cavity frequently manifests secondary effects of numerous systemic diseases and treatments during aging. The most relevant signs are a higher susceptibility to caries associated with xerostomia, periodontal inflammation and fungal infections. In this scenario, life style, medical conditions and therapeutic regimens, often concomitant in older persons, represent the major determi-

*Address correspondence to this author at the University of Milan, Department of Human Morphology and Biomedical Sciences "Città Studi"- LITA, Extracellular Matrix Laboratory, Via Fratelli Cervi 93, 20090 Segrate Milano, Italy; Tel: +3902 50330462; Fax: +39 02 50330452;

E-mail: nicoletta.gagliano@unimi.it nant. Moreover, poor oral health care, and dental or periodontal chronic infections could mediate weight change through alterations in diet and nutritional status, and also favour systemic effects [1-3].

Although a considerable amount of data are available regarding the effects of aging on the main tissues of the oral cavity, such as teeth, salivary glands, and periodontium [4, 5], very few studies investigated the age-related changes involving the gingiva [6].

A variety of age-related periodontal changes occur and, although controversial, earlier studies on aging gingiva showed that the metabolism of both collagen and noncollagenous proteins decreases with increasing age [7]. Agedependent modifications were also found in the fibroblast size [8], and in the mitotic activity of both gingival fibroblasts and epithelial cells $[9,10]$.

Qualitative and quantitative changes in gingival connective tissue are prominent features of periodontal diseases. A disturbance in connective tissue homeostasis, mainly involving interstitial collagen as the major structural protein in gin- 
gival connective tissue, has been suggested as one of the most relevant events occurring in the development of druginduced gingival overgrowth (GO), a pathology characterized by increased accumulation of interstitial collagen in the gingival connective compartment [11-13].

As previously reported, older people are susceptible to a higher incidence of multiple chronic disease and conditions compared with younger adults. As a consequence, they consume a greater number of prescription and non-prescription medications and, due to the age-associated changes of pharmacokinetic and pharmacodynamic properties, the elderly population is at greatest risk for medication-related problems that may very likely affect gingival connective homeostasis $[14,15]$.

Since the overall age-related gingival connective tissue modifications have still not been fully described [16], in the present study we combined different analytical approaches aimed at characterizing the complex mechanisms that control collagen turnover in aging gingival fibroblasts. In particular we aimed at: i) analyzing collagen content in young and aging gingivae, and the complex regulatory mechanisms that control collagen turnover in aging gingival fibroblasts using morphological and molecular approaches. ii) analyzing the effect of cyclosporin A on collagen turnover mechanisms in healthy aged gingival fibroblasts, compared to young ones in order to understand if older people are able to maintain tissue homeostasis or are more susceptible to the development of drug-induced gingival overgrowth.

This information might contribute to a better understanding of some of the modifications which gingiva undergoes with aging, providing a baseline to appraise the quantitative pathological changes of collagen turnover in disease states, and to understand the potential effect of pharmacologic treatment on gingival connective tissue.

\section{MATERIALS AND METHODOLOGY}

Experimental model: Gingival biopsies were obtained from 5 young (mean age $36 \pm 9.23,4$ females and 1 male) and 7 aging subjects (mean age $70.17 \pm 10.63,4$ females and 3 males). All subjects were no smokers, had clinically normal gingiva, with no signs of overt inflammation, hyperplasia and no history of drug use associated with GO, without any systemic health problems. Health, drugs, alcohol abuse and smoking histories were collected to exclude patients with known clinical situation able to affect the gingival status. They gave their informed consent to the biopsy, which was obtained from attached gingiva under local anaesthesia during minor oral surgical procedures. Each gingival biopsy was divided into two fragments: one fragment was processed for morphologic analysis and one fragment for cell culture evaluations.

Histochemistry and image analysis: Immediately after surgery, each gingival biopsy fragment was fixed in $10 \%$

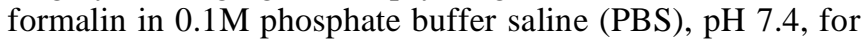
5 hours at room temperature, routinely dehydrated, paraffin embedded, and serially sectioned (thickness $5 \mu \mathrm{m}$ ). Sections were stained with freshly made haematoxylin-eosin to evaluate the tissue morphology. To specifically stain collagen, slides were deparaffinized and immersed for 30 minutes in saturated aqueous picric acid containing $0.1 \%$ Sirius red F3BA (Sigma, Italy).

All of the Sirius red-stained sections were analyzed by light microscopy and the images were captured and digitized using an image analysis system with specific software (Bio Image Analyzer, ICH, Italy). This software automatically selects the collagenous portion on the basis of similarities in the colour of adjacent pixels, based on a RGB system. Tissue COL content is expressed by a fibrosis index $(\%)$, indicating the ratio of the mean Sirius red-stained surface to the whole area of the section.

Cell culture: Human gingival fibroblasts were obtained from the young and aging subjects. Gingival biopsies were washed with sterile PBS, plated in T-25 flasks, incubated in DMEM supplemented with $10 \%$ heat-inactivated fetal bovine serum (FBS) and antibiotics $(100 \mathrm{U} / \mathrm{mL}$ penicillin, 0.1 $\mathrm{mg} / \mathrm{mL}$ streptomycin) at $37^{\circ} \mathrm{C}$ in a humidified atmosphere containing $5 \% \mathrm{CO}_{2}$. When fibroblasts grew out from the explant, they were trypsinized ( $0.25 \%$ trypsin- $0.2 \%$ EDTA) for secondary cultures. Viability was assessed by the Trypan blue exclusion method. Confluent human gingival fibroblasts were used between the fourth and fifth passage.

Molecular evaluations were done on fibroblasts cultured for $72 \mathrm{~h}$, using duplicate cultures for each sample.

Treatment of gingival fibroblasts with CsA: When human gingival fibroblasts grew to confluence in T-75 flasks, the culture medium was replaced with serum-free DMEM containing 2 doses of CsA (252 and $800 \mathrm{ng} / \mathrm{ml}$ ) dissolved in a vehicle $(0.008 \%$ ethanol, $0.0016 \%$ Tween-20). The lower dose corresponds to the whole blood trough levels of CsA. The higher dose was reported to induce modifications of proliferation and ECM turnover, and to affect collagen homeostasis in gingival fibroblasts obtained from young healthy subjects [12]. The cultures were then incubated at $37^{\circ} \mathrm{C}$ for $72 \mathrm{~h}$. To assess CsA effect, cultured fibroblasts of each sample incubated in CsA vehicle served as controls $(\mathrm{VH})$. At the established intervals of time the cell culture supernatants were collected and fibroblasts were washed in PBS, trypsinized and harvested by centrifugation (100 xg, 5 $\min )$.

Real-time RT-PCR: Total RNA was isolated by a modification of the acid guanidinium thiocyanate-phenolchloroform method (Tri-Reagent, Sigma, Italy). One $\mu \mathrm{g}$ of total RNA was reverse-transcribed in $20 \mu \mathrm{L}$ final volume of reaction mix (Biorad, Italy). mRNA levels of collagens type I (COL-I), long lysyl hydroxylase 2 (LH2b), matrix metalloproteinase 1 (MMP-1), tissue inhibitor of MMP (TIMP-1), Secreted Protein Acidic and Rich in Cysteine (SPARC), transforming growth factor- $\beta 1$ (TGF- $\beta 1$ ) were assessed.

The primers sequences, designed with Beacon Designer 6.0 Software (BioRad, Italy), were the following: GAPDH: sense CCCTTCATTGACCTCAACTACATG, antisense TG GGATTTCCATTGATGACAAGC; COL-I: sense CGAC CTGGTGAGAGAGGAGTTG, anti-sense AATCCATCCAGACCATTGTGTCC; MMP-1: sense CGGATACCCCA AGGACATCTACAG, antisense GCCAATTCCAGGAAA GTCATGTGC; TGF- $\beta 1$ : sense GTGCGGCAGTGGTTG AGC, antisense GGTAGTGAACCCGTTGATGTCC; TIMP-1: sense GGCTTCTGGCATCCTGTTGTTG, an- 
tisense AAGGTGGTCTGGTTGACTTCTGG; LH2b: sense CCG GAAACATTCCAAATGCTCAG, antisense GCCAGAGGT CATTGTTATAATGGG; SPARC: sense GCGAGCTG GATGAGAACAACAC, antisense GTGGCAAAGAAGT GGCAGGAAG.

GAPDH was used as endogenous control to normalize the differences in the amount of total RNA in each sample. Amplification reactions were conducted in a 96-well plate in a final volume of $20 \mu \mathrm{L}$ per well containing $10 \mu \mathrm{L}$ of $1 \mathrm{x}$ SYBR Green Supermix (BioRad, Italy), $2 \mu \mathrm{L}$ of template, 300 pmol of each primer, and each sample was analysed in triplicate. The cycle threshold $(\mathrm{Ct})$ was determined and gene expression levels relative to that of GAPDH were calculated by the $2^{-\Delta \Delta \mathrm{Ct}}$ method, according to Livak and Schmittgen [17].

Slot blot: Cell culture media from young and aging fibroblasts were concentrated 20-fold with Centricon 10 columns (Amicon Y10, Millipore, Italy). Protein content was determined by a standardized colorimetric assay (DC Protein Assay, Bio Rad, Italy); $20 \mu \mathrm{g}$ of total protein per sample in a final volume of $200 \mu \mathrm{L}$ of Tris buffer saline (TBS) were spotted onto a nitrocellulose membrane in a Bio-Dot SF apparatus (Bio-Rad, Italy). Membranes were blocked for $1 \mathrm{~h}$ with 5\% skimmed milk in TBST (TBS containing $0.05 \%$ tween-20), $\mathrm{pH} 8$, and incubated for $1 \mathrm{~h}$ at room temperature in monoclonal antibody to COL-I (1:1000 in TBST) (Sigma, Italy) or to COL-III (1:2000 in TBST) (Sigma, Italy). After washing, membranes were incubated in HRP-conjugated rabbit anti-mouse serum (1:80,000 in TBST) (Sigma, Italy) for $1 \mathrm{~h}$. Immunoreactive bands revealed by the Amplified Opti-4CN substrate (Amplified Opti-4CN, Bio Rad, Italy) were scanned densitometrically (UVBand, Eppendorf, Italy).

MMP-1 protein expression in VH and CsA treated fibroblasts was assessed by slot blot. The membranes were incubated overnight at $4{ }^{\circ} \mathrm{C}$ in monoclonal antibody to MMP-1 (1 $\mu \mathrm{g} / \mathrm{ml}$ in TBST, Calbiochem) and, after washing, in HRPconjugated rabbit anti-mouse serum (1:80,000 dilution, Sigma).

SDS-zymography: Concentrated culture media were mixed 3:1 with sample buffer (containing 10\% SDS). Samples (five $\mu \mathrm{g}$ of total protein per sample) were run under non-reducing conditions without heat denaturation onto $10 \%$ polyacrylamide gel (SDS-PAGE) co-polymerized with 1 $\mathrm{mg} / \mathrm{mL}$ of type I gelatin. The gels were run at $4^{\circ} \mathrm{C}$. After SDS-PAGE, the gels were washed twice in $2.5 \%$ Triton X100 for 30 min each and incubated overnight in a substrate buffer at $37^{\circ} \mathrm{C}$ (Tris- $\mathrm{HCl} 50 \mathrm{mM}, \mathrm{CaCl}_{2} 5 \mathrm{mM}, \mathrm{NaN}_{3} 0.02 \%$, $\mathrm{pH}$ 7.5). The matrix metalloproteinase (MMP) gelatinolytic activity was detected after staining the gels with Coomassie brilliant blue R250, as clear bands on a blue background.

Western blot: Concentrated culture media (5 $\mu \mathrm{g}$ of total proteins) were diluted in SDS-sample buffer, loaded on $10 \%$ SDS-polyacrylamide gel, separated under reducing and denaturing conditions at $80 \mathrm{~V}$ according to Laemmli, and transferred at $90 \mathrm{~V}$ to a nitrocellulose membrane in $0.025 \mathrm{M}$ Tris, $192 \mathrm{mM}$ glycine, $20 \%$ methanol, $\mathrm{pH}$ 8.3. After electroblotting, the membranes were air dried and blocked for 1 hour. After being washed in TBST (TBS/Tween-20 0.05\%), membranes were incubated for $1 \mathrm{~h}$ at room temperature in mono- clonal antibody to SPARC (1:100 in TBST, Novocastra Laboratories) and, after washing, in HRP-conjugated rabbit anti-mouse serum (1:6000 dilution, Sigma, Italy). Immunoreactive bands were revealed using the Opti-4CN substrate Bio Rad.

Statistical analysis: All amplifications were run in triplicate, and all of the evaluations were repeated two times. To compare the young and aging untreated fibroblasts, data were analyzed by an unpaired t-test, and the age was the independent variable. The hypothesis was 2-tailed. To assess the effects of aging and CsA administration, data were analyzed by 2-way ANOVA. $p$ values less than 0.05 were considered significant. A post hoc assessment of the power of the t-test [18] and the variance test made using the noncentral F-distribution [19] was performed.

\section{RESULTS}

Our first aim was to characterize collagen content and turnover in both gingival tissues and cultured gingival fibroblasts obtained from healthy young and aging subjects.

Morphological and quantitative image analysis: Under light microscopy haematoxylin-eosin stained sections of young and aging samples appeared similar (Fig. 1a, b). In the epithelium of the two experimental groups we observed a similar number of the living cell layers, excluding the presence of acanthosis. The rete peg was similarly prolonged in both experimental groups, and regularly elongated. The connective tissue included a similar amount of collagen fiber bundles running in all directions, with some inflammatory cell infiltration which tended to be more abundant in some of the aging gingival fragments.

Sirius red stained the collagen specifically and computerized analysis of sections of gingiva from young and aging subjects indicated that the fibrosis index was, respectively, $83.5 \% \pm 6.29$ and $82.5 \% \pm 4.74$ (Fig. 1c, d).

Gene expression: mRNA levels are shown in Fig. (2a). COL-I gene expression decreased in aging fibroblasts compared to the young ones $(\mathrm{p}<0.05)$. A tendency to decrease was observed for TIMP-1 ( $p=0.068)$, while MMP-1 and TGF- $\beta 1$ mRNA levels were unchanged ( $p$ ns). LH $2 b$ mRNA levels in aging fibroblasts were unaffected. Interestingly, if we consider the LH2b/COL-I mRNA ratio, there was a significant increase $(p<0.05)$ of this ratio in aging fibroblasts compared to young ones.

COL protein levels: COL-I and COL-III protein levels were assessed in fibroblast supernatants by slot blot. Densitometric scanning of immunoreactive bands showed that COL-I and COL-III content was not affected by aging (data not shown).

COL degradation: MMP-1 and MMP-2 protein levels in young and aging fibroblast supernatants is presented in Fig. (2b and 2c). The densitometric analysis of lytic bands identified as MMP-1 revealed similar levels of interstitial collagenase in the supernatants obtained from young and aging subjects (Fig. 2b, c). This result was confirmed by slot blot analysis (data not shown). Also MMP-2 protein levels seems unaffected by aging (Fig. 2b, c). 

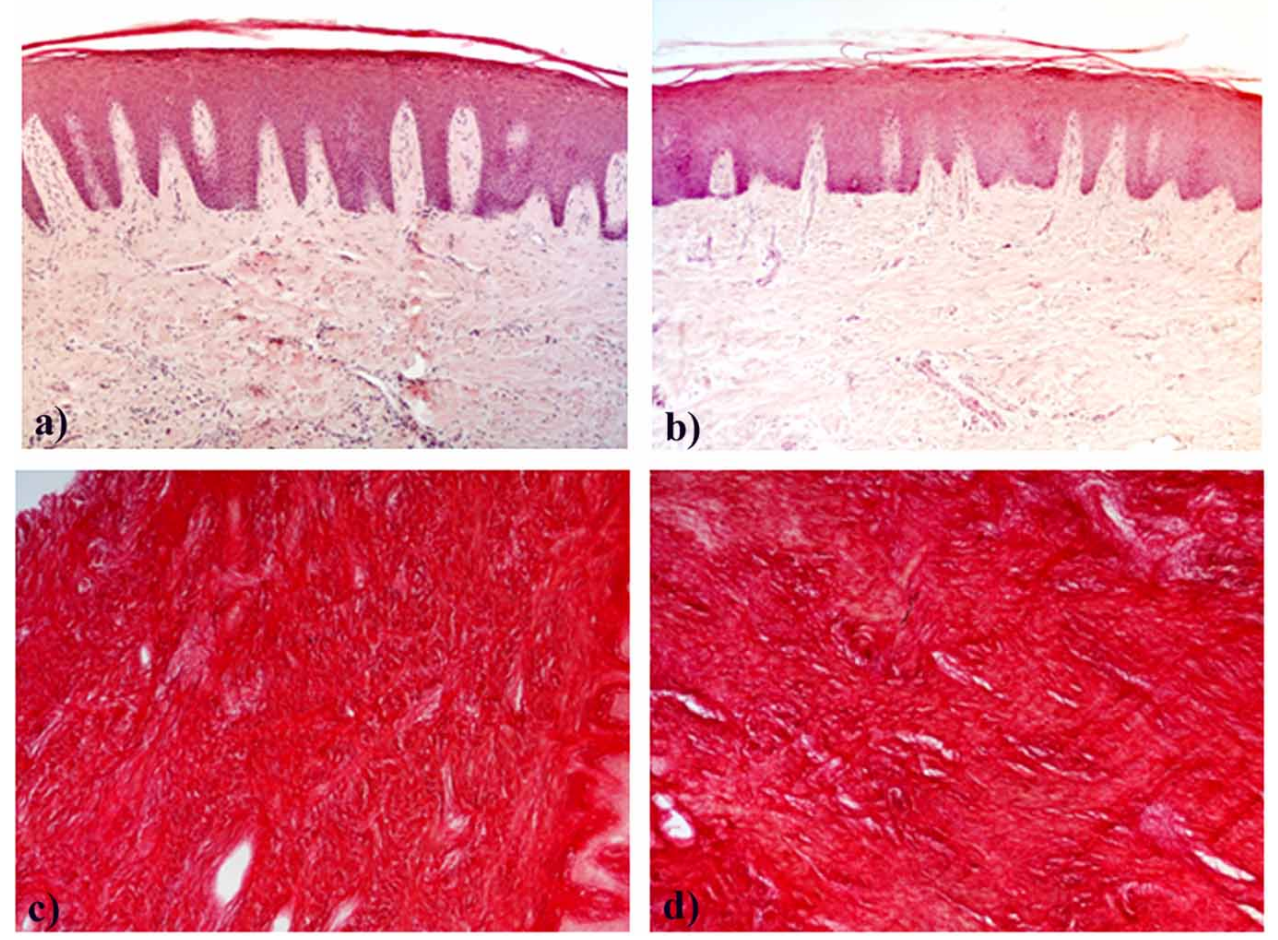

Fig. (1). Microphotographs of young and aging gingiva paraffin-embedded sections stained with hematoxylin-eosin (a, b) or with Sirius red $(\mathbf{c}, \mathbf{d})$. a and c: young gingiva; $\mathrm{b}$ and $\mathrm{d}$ : aging gingiva. Original magnification: 10X.

SPARC expression: SPARC mRNA levels tended to be lower in aging compared to young fibroblasts $(0.46 \pm 0.08$ and $0.96 \pm 0.079$, respectively, p ns). SPARC protein levels, however, were not significantly affected by the aging process (young $195095 \pm 110644$, aging $170894 \pm 173511$, p ns).

Post hoc power assessment of the statistical test revealed that the power was $>0.90$ for COL-I and SPARC gene expression, indicating that the analysis had not type II errors. The power was comprised between 0.4 and 0.6 for MMP-1 mRNA, TIMP-1 mRNA and LH2b/COL-I mRNA ratio, and was $<0.2$ for LH2b and TGF- $\beta 1$ mRNA levels, indicating that the analyses had a type II errors.

\section{Effect of CsA on Collagen Turnover in Young and Aging Fibroblasts}

Our second aim was to characterize if collagen turnover was affected by CsA administration at a different extent in cultured gingival fibroblasts obtained from healthy young and aging subjects.

Gene expression: COL-I gene expression (Fig. 3a) was not affected by CsA administration both in young and in aging fibroblasts $(\mathrm{p}$ [age $]<0.0005, \mathrm{p}$ [treatment $]=\mathrm{ns}, \mathrm{p}$ [interaction $]=n s)$. A similar pattern was observed for MMP-1 $(\mathrm{p}[$ age $]=\mathrm{ns}, \mathrm{p}[$ treatment $]=\mathrm{ns}, \mathrm{p}$ [interaction $]=\mathrm{ns})($ Fig. 3b), TIMP-1 (p [age] $<0.05$, p [treatment] = ns, p [interaction] $=\mathrm{ns})($ Fig. 3c), TGF- $\beta 1(\mathrm{p}$ [age] $=\mathrm{ns}, \mathrm{p}$ [treatment $]=$ $\mathrm{ns}, \mathrm{p}$ [interaction] $=\mathrm{ns})($ Fig. 3d), LH2b (p [age] $=\mathrm{ns}, \mathrm{p}$ [treatment $]=n s, p$ [interaction] $=n s)($ Fig. 3e), and SPARC mRNA levels $(\mathrm{p}$ [age] $=\mathrm{ns}, \mathrm{p}$ [treatment $]=\mathrm{ns}, \mathrm{p}$ [interaction] $=$ ns) (Fig. 3f).

COL protein levels: COL-I protein levels were assessed in fibroblast supernatants by slot blot. Densitometric scan- ning of immunoreactive bands showed that COL-I levels tended to be higher in CsA-treated young fibroblasts, and were not modified by CsA in aging fibroblasts (Fig. 4 a).

COL degradation: MMP-1 protein levels in young and aging fibroblast supernatants is presented in Fig. 4 b. MMP1 protein levels tended to be down-regulated in CsA-treated young fibroblasts, compared to $\mathrm{VH}$. In aging fibroblasts we observed a wide interindividual variation and a tendency to decrease after $800 \mathrm{ng} / \mathrm{ml} \mathrm{CsA}$, compared to $\mathrm{VH}$ (p [age] = $\mathrm{ns}, \mathrm{p}$ [treatment $=\mathrm{ns}, \mathrm{p}$ [interaction] $=\mathrm{ns})$. MMP-2 activity was unchanged after CsA administration in both young and aging fibroblasts $(\mathrm{p}$ [age] $=\mathrm{ns}, \mathrm{p}$ [treatment] $=\mathrm{ns}, \mathrm{p}$ [interaction]= ns) (data not shown).

SPARC expression: SPARC protein levels were unchanged by CsA administration in both young and aging gingival fibroblasts $(\mathrm{p}$ [age] $=\mathrm{ns}, \mathrm{p}$ [treatment] $=\mathrm{ns}, \mathrm{p}$ [interaction $]=n s)($ Fig. 4c), as observed for SPARC mRNA levels $(\mathrm{p}[$ age $]=\mathrm{ns}, \mathrm{p}[$ treatment $]=\mathrm{ns}, \mathrm{p}[$ interaction $]=\mathrm{ns})$.

Post hoc power assessment of the statistical test revealed that the power was comprised between 0.88 and 0.99 for COL-I mRNA and protein levels, MMP-1 mRNA and protein levels, and TIMP-1 mRNA levels, suggesting that the analysis had not type II errors. For the remnant evaluations, the power was comprised between 0.30 and 0.40 , indicating that the analyses had type II errors.

\section{DISCUSSION}

Chronic diseases are common among older people and increase the necessity for multiple medications. Polipharmacotherapy, together with the well established changes of the pharmacokinetic processes, render the elderly more susceptible to the adverse effects of drugs [20]. 
a)

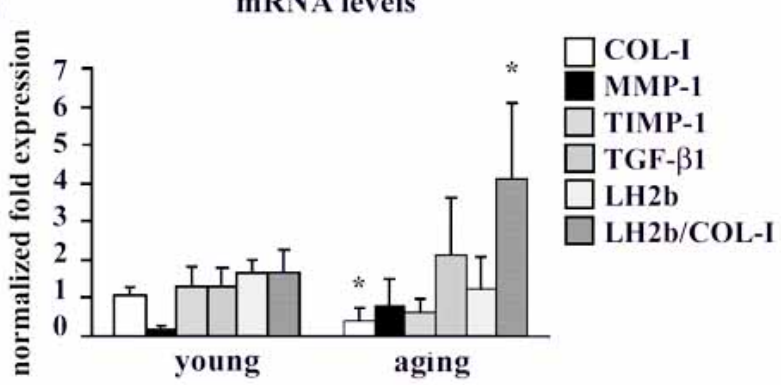

b)

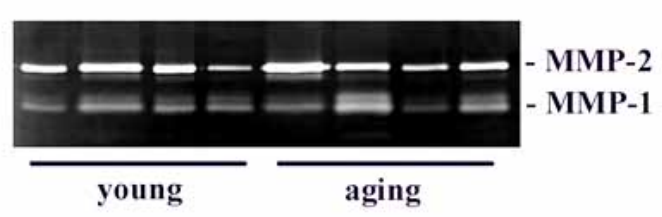

c)

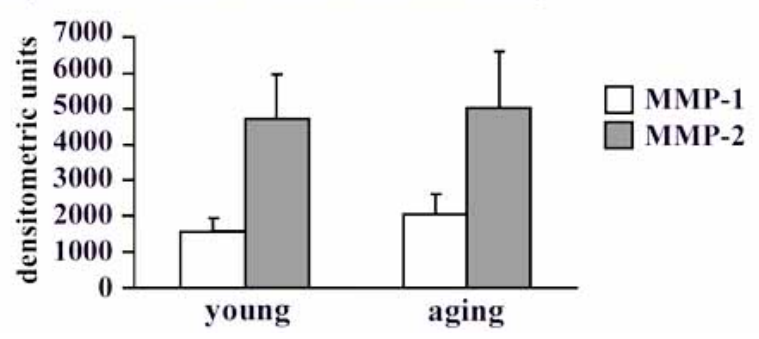

Fig. (2). a) Bar graphs showing COL-I, MMP-1, TIMP-1, TGF- $\beta 1$ and LH2b mRNA levels, and the LH2b/COL-I mRNA ratio in young and aging cultured fibroblasts. Changes in mRNA are normalized on GAPDH gene expression. Values are means \pm SD for duplicate samples. * indicates a significant difference $(\mathrm{p}<0.05$, unpaired Student's t test). b) Representative gelatin zymogram of MMPs in serum-free, conditioned human gingival fibroblast supernatants from young and aging gingival. The lytic bands weighing $66 \mathrm{kDa}$ and in the $60 / 50 \mathrm{kDa}$ region correspond to proMMP-1 and proMMP-2, respectively. c) Bar graphs showing proMMP-1 and proMMP-2 levels in fibroblast serum-free conditioned media after densitometric analysis of lytic bands following SDS-zymography. Data are expressed as densitometric units and are means \pm SD.

Periodontal tissue is susceptible to a range of systemic medications able to promote unwanted effects (e.g. GO), and the gingiva may be therefore a target of adverse reactions, particularly in the elderly.

Collagen is the main component of gingival connective compartment. Its turnover is a vital step in the tissue remodelling that accompanies physiological and pathological processes, and its age-dependent imbalance is a hallmark of the aging of various organs, i.e. heart and the kidney [21, 22], but very few data are available regarding the gingiva.

Gingival connective tissue undergoes a rapid rate of collagen turnover [23], therefore all of the mechanisms involved (collagen synthesis, maturation, and degradation) are of considerable importance in the net collagen steady-state, in which MMPs play a pivotal role [24]. MMPs with collagenase and gelatinase activities are secreted in the extracellu- lar space as zymogens and are activated by proteolytic cleavage; their activity is closely regulated and under pathological conditions any mismatch could result in excessive ECM accumulation or degradation.

Interstitial collagenase or MMP-1 cleaves the native triple helical region of interstitial COL into characteristic 3/4and 1/4-collagen degradation fragments [25], allowing its further digestion by less specific proteinases, leading to complete digestion of fibrillary COL.

The extracellular activity of MMPs is tightly regulated at various points, including inhibition by TIMPs, among which TIMP- 1 and 2 are the most important since they inhibit the active form of all MMPs, forming inactive 1:1 stoichiometric TIMP-1/MMP-1 complexes [26, 27].

During COL maturation, post-translational hydroxylation of COL lysine residues, accomplished by lysyl hydroxylase $(\mathrm{LH})$ is a key mechanism influencing collagen biosynthesis and extracellular matrix stability [28]. Two alternatelyspliced forms of LH2 exist, the long (LH2b) and the short (LH2a) ones [28]. We investigated LH2b gene expression, since it is the major form expressed in all tissues and is generally overexpressed in fibrotic processes [29]. LH2b, in fact, is responsible for the over-hydroxylation of the COL telopeptides, forming COL pyridinoline cross-links, thus contributing to unwanted COL accumulation [29].

Our first aim was to characterize collagen turnover pathways in untreated aging gingival fibroblasts, compared to young ones. COL content, as shown by the fibrosis index and protein analysis results, is similar in young and aging subjects. Moreover, our results show unchanged MMP-1 as well as TIMP-1 mRNA levels, and similar MMP-1 and MMP-2 protein levels, suggesting that gingival collagen content is not regulated at the level of its degradation. This was consistent by the increased LH2b/COL-I mRNA ratio (indicating the gene expression of $\mathrm{LH} 2 \mathrm{~b}$ relative to COL-I). Although the LH2b/COL-I mRNA ratio increased due to decreased COL-I mRNA levels with age, we can hypothesize that the newly synthesized collagen could be more susceptible to be cross-linked in aged compared to young gingival fibroblasts, and therefore less susceptible to degradation [30]. This hypothesis is consistent with the finding of COL-I mRNA levels down-regulation in aging fibroblasts, of a similar fibrosis index and interstitial collagen content in both the experimental groups, together with a similar MMPs activity, suggesting that in young and aging gingival collagen regulation may be very likely dependent in part on the maturation pathways carried out by post-translational modifications such as collagen crosslinking.

The overall balance of COL turnover is controlled also by TGF- $\beta 1$, a multifunctional cytokine known to be involved in both healing and fibrogenic processes, being capable of regulating cell proliferation and differentiation as well as directly activating gene expression for the synthesis of ECM components [31]. Also TGF- $\beta 1$ gene expression was unchanged in aging fibroblasts, suggesting a similar tone of TGF- $\beta 1$ influencing collagen turnover.

SPARC is an important multifunctional glycoprotein that modulates cellular interactions with ECM by its binding to structural matrix proteins [32]. In adults, the expression of 

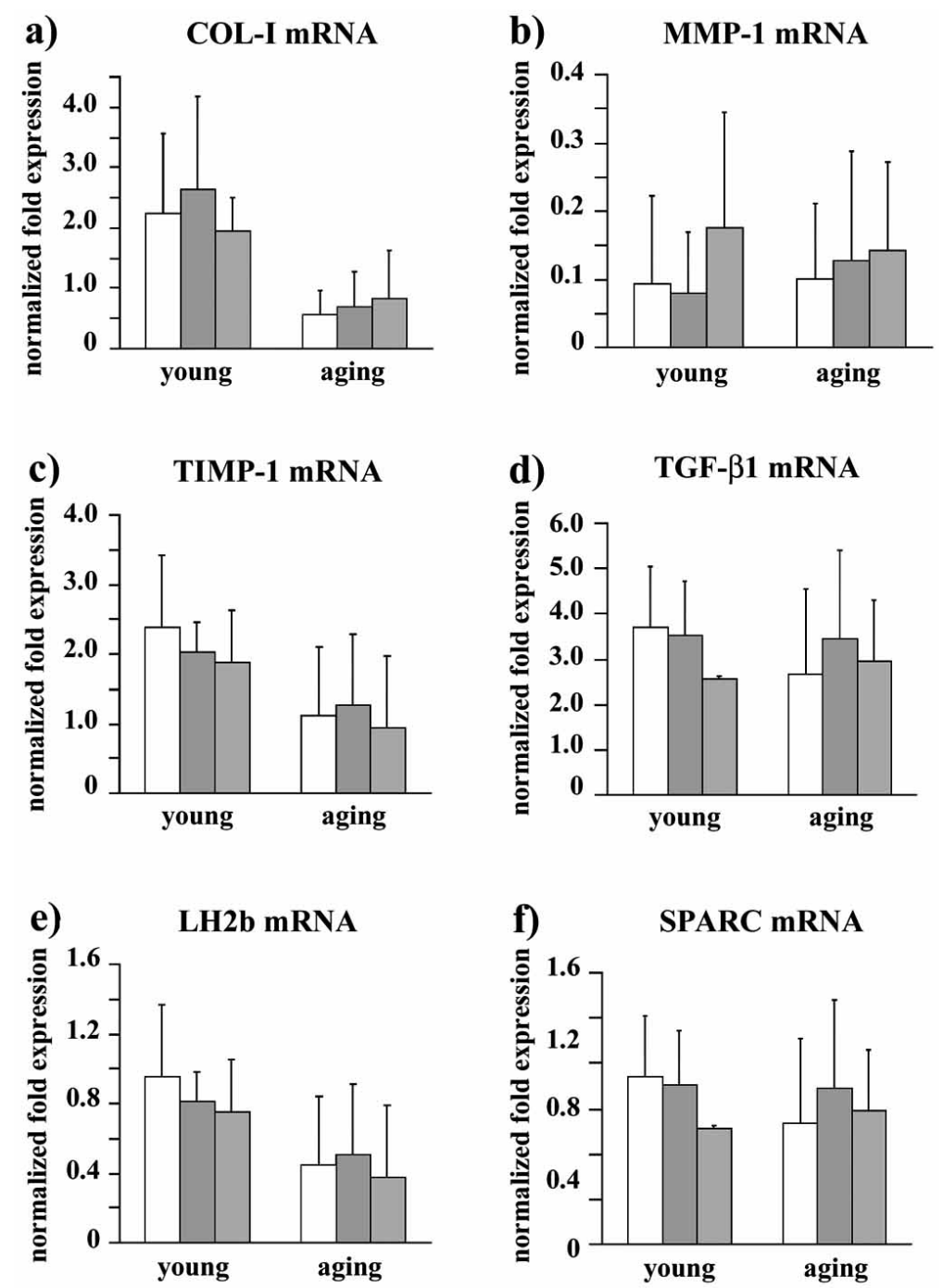

\begin{abstract}
VH
CsA $250 \mathrm{ng} / \mathrm{ml}$

$\operatorname{CsA} 800 \mathrm{ng} / \mathrm{ml}$

Fig. (3). Bar graphs showing mRNA levels for COL-I (a), MMP-1 (b), TIMP-1 (c), TGF- $\beta 1$ (d), LH2b (e), and SPARC (f) in VH and CsAtreated gingival fibroblasts obtained from young and aging healthy donors. mRNA are normalized on GAPDH gene expression. Values are means \pm SD for duplicate samples. VH: vehicle.
\end{abstract}

SPARC is limited to tissues undergoing repair or remodelling, and healthy gingival connective tissue is a remodelling tissue with high turnover [33,34]. Since SPARC protein levels seem not significantly affected by the aging process, we can hypothesize that gingival connective tissue remodelling occurs at a similar extent in young and aged people.

Our second aim was to analyze the effect of CsA on collagen turnover in healthy aging gingival fibroblasts, compared to young ones. Our results show that collagen turnover pathways are similarly affected by CsA treatment in young and aging gingival fibroblasts. Aging induced the increase of collagen protein levels and TIMP-1 mRNA down-regulation, but we did not find any statistical difference due to agingCsA interaction, and therefore the described differences between young and aging CsA-treated fibroblasts are due to aging itself and not to CsA administration. Considered as a whole, these findings suggest that young and aging gingival fibroblasts respond to CsA administration at the same extent.

\section{CONCLUSION}

Considered as a whole, the present study offers new insights on the relationship between aging and gingival connective tissue homeostasis. Our results show that in the absence of any critical disorder in healthy aged people, gingival connective tissue is characterized by a similar collagen content and turnover, suggesting that in the senescent phenotype, the complex machinery that controls collagen turnover is balanced. We hypothesized that, since in the elderly the defence machinery against the xenobiotic is weakened, and since the elderly use a higher number of medications, they are more susceptible to drug effects due to diminished ability to maintain tissue homeostasis. In particular, interstitial collagen is the main component of gingival connective tissue and its turnover is the target of several drugs leading to GO, such as CsA. Although collagen turnover is the main target of CsA in gingival fibroblasts, our results show that during aging, CsA administration is not related to relevant collagen 
a)

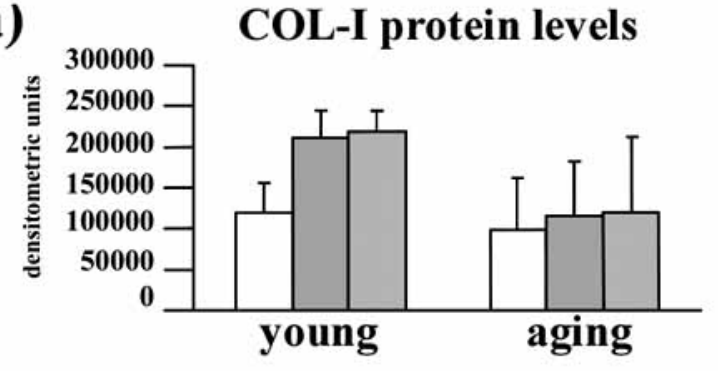

b)

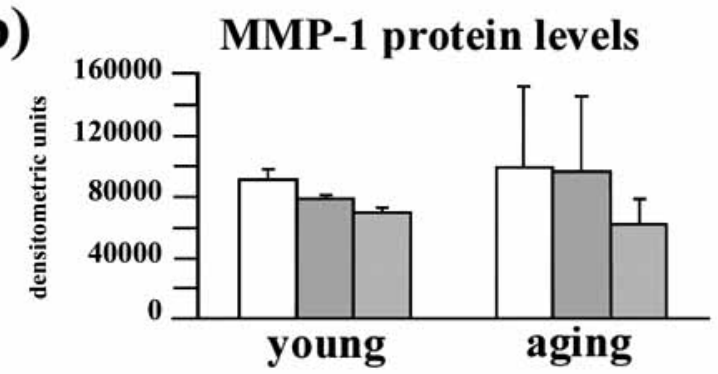

c)

)

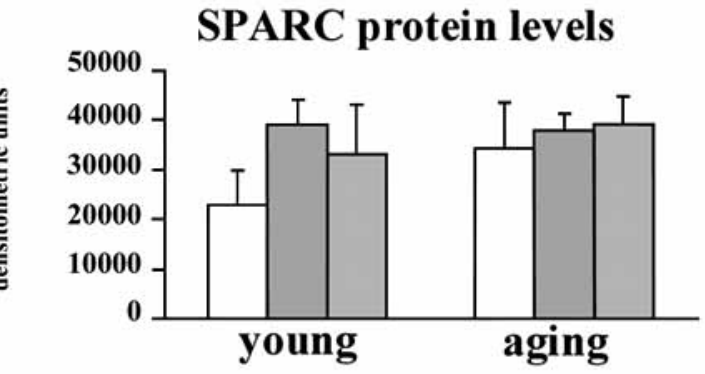

VH

CsA $250 \mathrm{ng} / \mathrm{ml}$

CsA $800 \mathrm{ng} / \mathrm{ml}$

Fig. (4). Bar graph presenting COL-I (a), MMP-1 (b), and SPARC (c) protein levels $\mathrm{VH}$ and CsA-treated gingival fibroblasts obtained from young and aging healthy donors, assessed by slot blot or Western blot analysis, as described in the Material and Methods section. Data are reported as densitometric units after scanning of the immunoreactive bands. Values are means \pm SD for duplicate samples. VH: vehicle.

turnover modifications, and that CsA treatment affects collagen turnover pathways at a similar extent in young and aging gingival fibroblasts.

The post hoc assessment of the power of the study revealed that in some occasions a type II error was committed. As a consequence, this study presents a limit due to the number of the analyzed subjects: the lack of statistical significance should be taken with caution. However, our results concerning the effect of CsA administration on young and aging healthy gingival fibroblasts are consistent since COL-I and MMP-1 are the main targets of CsA, and the power of the study for these two parameters was $>0.88$.

\section{ACKNOWLEDGEMENTS}

We would like to thank Dr. Marco Giorgio Bianchi (BioRad Laboratories System Specialists - Gene eXpr. Division) for excellent technical assistance, and the Ariel Foundation for financial support.

\section{REFERENCES}

[1] Saunders MJ. Functional ability and oral health: reintegrating mouth and body. JAGS 2001; 49: 1000-1.

[2] Weyant RJ, Newman AB, Kritchevsky SB, et al. Periodontal disease and weight loss in older adults. J Am Geriatr Soc 2004; 52: 547-53.

[3] Bretz WA, Weyant RJ, Corby PM, et al. Systemic inflammatory markers, periodontal diseases, and periodontal infections in an elderly population. J Am Geriatr Soc 2005; 53: 1532-37.

[4] Baum BJ, Ship JA. The oral cavity. In: Hazzard WR, Andres R, Bierman EL, Blass JP, Eds. Principle of geriatric medicine and gerontology, $2^{\text {nd }}$ ed. New York: McGraw-Hill. 1990; pp. 413-21.

[5] Benatti BB, Silvério KG, Casati MZ, Sallum EA, Nociti FH Jr. Influence of aging on biological properties of periodontal ligament cells. Connect Tissue Res 2008; 49: 401-8.

[6] Van der Velden U. Effect of age on the periodontium. J Clin Periodontol 1984; 11: 281-94.

[7] Johnson BD, Page RC, Narayanan S, Pieters HP. Effects of donor age on protein and collagen synthesis "in vitro" by human diploid fibroblast. Lab Invest 1986; 55: 490-6.

[8] Amstad-Jossi M, Schroeder HE. Age related alterations of periodontal structure around the cemento-enamel junction and of the gingival connective tissue composition in germ-free rats. J Periodont Res 1987; 13: 76-9.

[9] Karring T, Loe $\mathrm{H}$. The effect of age on mitotic activity in rat oral epithelium. J Periodont Res 1973; 8: 164-70.

[10] Celenligil-Nazliel H, Ayhan A, Ruacan S. The effect of age on proliferating cell nuclear antigen expression in oral gingival epithelium of healthy and inflamed human gingiva. J Periodontol 2000; 71: 1567-74.

[11] Hassell TM, Hefti AF. Drug-induced gingival overgrowth: old problem, new problem. Crit Rev Oral Biol Med 1991; 2: 103-137.

[12] Gagliano N, Moscheni C, Dellavia C, et al. Effect of cyclosporin A on human gingival fibroblast collagen turnover in relation to the development of gingival overgrowth: an in vitro study. Biomed Pharmacother 2004; 58: 231-8.

[13] Seymour RA. Effects of medications on the periodontal tissues in health and disease. Periodontol 2000 2006; 40: 120-29.

[14] Ciancio S. Medications' impact on oral health. JADA 2004; 135: 1440-48.

[15] Turnheim K. Drug therapy in the elderly. Exp Gerontol 2004; 39: 1731-38.

[16] Gogly B, Godeau G, Gilbert S, et al. Morphometric analysis of collagen and elastic fibers in normal skin and gingival in relation to age. Clin Oral Invest 1997; 1: 147-52.

[17] Livak KJ, Schmittgen TD. Analysis of relative gene expression data using real-time quantitative PCR and the 2(- delta deltaC(T)) method. Methods 2001; 25: 402-8.

[18] Lenth RV. Java Applets for Power and Sample Size [Computer software]. Retrieved July, $3^{\text {rd }}, \quad 2009$; from http://www.stat.uiowa.edu/ rlenth/Power.

[19] Soliani L. Manuale di Statistica per la Ricerca e la Professione Statistica Univariata e Bivariata Parametrica e Non Parametrica per le Discipline Ambientali e Biologiche. Available at: www.dsa.unipr.it/soliani.

[20] McLean A, Le Couteur DG. Aging biology and geriatric clinical pharmacology. Pharmacol Rev 2004; 56: 163-4.

[21] Annoni G, Luvarà G, Arosio B, et al. Age-dependent expression of fibrosis-related genes and collagen deposition in the rat myocardium. Mech Ageing Dev 1997; 101: 52-72.

[22] Gagliano N, Arosio B, Santambrogio D, et al. Age-dependent expression of fibrosis-related genes and collagen deposition in rat kidney cortex. J Gerontol 2000; 55: B365-72.

[23] Sodek J. A comparison of the rates of synthesis and turnover of collagen and non-collagen proteins in adult rat periodontal tissues and skin using a microassay. Arch Oral Biol 1977; 22: 655-65.

[24] Kinane DF. Regulators of tissue destruction and homeostasis as diagnostic aids in periodontology. Periodontol 2000 2000; 24: 21525 . 
[25] Sakai T, Gross J. Some properties of the products of reaction of tadpole collagenase with collagen. Biochemistry 1967; 6: 518-28.

[26] Woessner JF. Matrix metalloproteinases and their inhibitors in connective tissue remodeling. FASEB J 1991; 5: 2145-54.

[27] Brew K, Dinakarpandian D, Nagase H. Tissue inhibitors of metalloproteinases: evolution, structure and function. Biochim Biophys Acta 2001; 1477: 267-83.

[28] Walker LC, Overstreet MA, Yeowell HN. Tissue-specific expression and regulation of the alternatively-spliced forms of lysyl hydroxylase 2 (LH2) in human kidney cells and skin fibroblasts. Matrix Biol 2005; 23: 515-23.

[29] Van der Slot AJ, Zuurmond A, van der Bogaerdt AJ, et al. Increased formation of pyridoline cross-links due to highetr telopeptide lysyl hydroxylase levels is a general fibrotic phenomenon. Matrix Biol 2004; 23: 251-57.
[30] Kagan H. Characterization and regulation of lysyl oxidase. In: Mecham RP, ed. Regulation of matrix accumulation, vol. 1. Orlando, Academic Press 1986; pp. 321-98.

[31] Border WA, Ruoslahti E. Transforming growth factor- $\beta$ in disease: the dark side of tissue repair. J Clin Invest 1992; 90: 1-7.

[32] Brekken RA, Sage EH. SPARC, a matricellular protein: at the crossroads of cell-matrix communication. Matrix Biol 2001; 19: 816-27.

[33] Porter PL, Sage EH, Lane TF, Funk SE, Gown AM. Distribution of SPARC in normal and neoplastic human tissue. J Histochem Cytochem 1995; 43: 791-800.

[34] Bartold PM, Walsh LJ, Narayanan AS. Molecular and cell biology of the gingiva. Periodontol 2000 2000; 24: 28-55.

(C) Gagliano et al.; Licensee Bentham Open.

This is an open access article licensed under the terms of the Creative Commons Attribution Non-Commercial License (http://creativecommons.org/licenses/by-nc/3.0/) which permits unrestricted, non-commercial use, distribution and reproduction in any medium, provided the work is properly cited. 